\title{
Foreword
}

\section{Sports Imaging: Plan Your Trip}

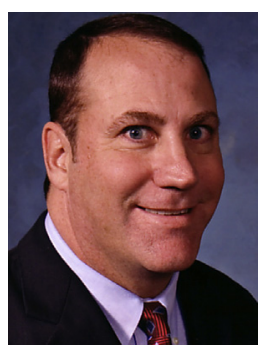

Mark D. Miller, MD Consulting Editor

You wouldn't go on a road trip without first looking at a map (or the twenty-first century equivalent: GPS). Likewise, you shouldn't plan surgery without first obtaining and reviewing relevant imaging studies. This issue of Clinics in Sports Medicine should be considered map-reading 101. I asked two of our very talented University of Virginia (UVA) musculoskeletal radiology orienteers, Drs Nicholas Nacey and Jennifer Pierce, to help sports surgeons plan future trips. They elected to start North and head South, beginning with the shoulder and proceeding to the foot. They also have highlighted a few road hazards, stress imaging, pediatric imaging, and ultrasound to keep us on the highway. As the Guest Editors point out, surgeons and radiologists enjoy a tremendous collaborative relationship at UVA that benefits us all. In addition to the monthly MRIArthroscopy correlation conference, we visit the reading room on a regular basis and often send our fellows over for a "road trip" to gain a better understanding of what lies ahead. I would encourage you to do the same, and spend some time picking up a few orienteering skills offered in this issue. Happy Trails!

Mark D. Miller, MD

Division of Sports Medicine Department of Orthopaedic Surgery University of Virginia, Charlottesville 400 Ray C. Hunt Drive Suite 330

Charlottesville, VA 22908-0159, USA

E-mail address: MDM3P@hscmail.mcc.virginia.edu 